\title{
Study of the relationship of electrical conductivity of porous moisture-saturated soils polluted with inorganic ecotoxicants
}

\author{
Sergey Prostov ${ }^{1}$ and Evgeniy Shabanov ${ }^{1, *}$ \\ ${ }^{1}$ T.F. Gorbachev Kuzbass State Technical University, 28, Str. Vesennyaya, 650000, Kemerovo, \\ Russia
}

\begin{abstract}
The relevance of the study of soils polluted with solutions of acids and alkalis is given in the article. The main theoretical dependences of the electrical conductive properties on the parameters of the soil body are considered. The database of the values of the parameters of the pore space of soils of the Kemerovo region - Kuzbass is given. The electrical properties of water with different salt content, water solutions with different content of acids and alkalis have been determined. A database for interpreting the results of experimental sounding when monitoring changes in the ratio of the pollutant content to the volume of the pore space, depending on the electrical resistivity, has been formed. The relationship between the electrical conductive properties of solutions of acids and alkalis and their content has been established. It was found that with an increase in the content of acids and alkalis in the solution, a monotonic increase in the electric resistivity occurs. It was found that with an increase in the content of acids and alkalis in the solution, a monotonic decrease in the resistivity occurs; this regularity is the physical basis of the method for diagnosing contaminated zones and monitoring the processes of their decontamination.
\end{abstract}

\section{Introduction}

At present, special attention is paid to environmental issues, since the environmental load resulting from the intense anthropogenic impact of various industries has increased [13]. In the industrialized regions of the Russian Federation, the issue of soil pollution is especially acute. Chemical pollution has the greatest impact on upsetting the biological balance of the soil. Its main sources are industry, agriculture and transport [4-6]. The impact of these human activities can lead to soil fatigue, a decrease in its fertility. Chemical pollutants in the soil from industrial activities can penetrate crops and then enter the organisms of animals and people. They can also have a direct impact on the pollution of surface and ground waters as a result of the leaching of harmful substances from the ground.

A significant part of ecotoxicants are electrically contrast agents. For example, all petroleum products exhibit pronounced dielectric properties, while phenol, formaldehyde,

* Corresponding author: evgenshab@mail.ru 
and acids are electrolytes and have low electrical resistance. Therefore, contaminated areas are anomalous electrically contrast zones that can be diagnosed by electrical prospecting, GPR and other geophysical methods.

Alkaline and acid batteries are actively used (automobile, underground railway transport, individual lamps, etc.) in the mining industry; during the maintenance of which there are electrolyte leaks, which are also ecotoxicants.

Active pollution of soils with alkaline and acid-containing ecotoxicants occurs when using underground leaching technologies, hydraulic fracturing, and storage of waste from processing plants.

Direct and indirect instrumental methods are used to monitor soil pollution. Direct methods (infrared spectrophotometry, ultraviolet luminescence, gas and gas-liquid chromatography) provide for the use of complex equipment, significant analysis duration and mandatory soil sampling, while the measurement error can reach $40-50 \%$. In connection with the above, the role of measuring the content of pollutants in the soil by an indirect geophysical method, by measuring the electrical parameters of the soil, increases.

\section{Research methods}

The basic physical prerequisite for the control method is that all acids and alkalis (electrolyte in the battery, etc.) exhibit electrical contrast and have a relatively low electrical resistance. The effective resistivity (ER) of these liquids varies in the range $\rho=$ $0.01-1 \mathrm{Ohm} \cdot \mathrm{m}$ depending on the content, while for natural mineral solutions this range is $\rho$ $=1-100 \mathrm{Ohm} \cdot \mathrm{m}$. The effective resistivity of a moisture-saturated rock, which is a threephase medium, is determined using the classical empirical dependence:

$$
\rho_{\mathrm{\kappa}}=\frac{\alpha K_{n}}{m^{\beta} W^{\gamma}} \rho_{\mathrm{B}},
$$

where $m$ - porosity (void content); $W$ - moisture saturation coefficient of pore space and cracks; $\rho_{\theta}$ - effective resistivity of pore filling solution, Ohm·m; $K_{n}, \alpha, \beta, \gamma-$ empirical parameters depending on the structural and textural features of the studied soils (rocks); $K_{n}$ - parameter that takes into account the surface conductivity of the clay microlayer on the pore surface; $\alpha$-parameter depending the deposit type; $\beta$-parameter determined by the pore space structure (mainly the tortuosity); $\gamma$ - parameter depending on the solution wettability of the pore surface.

The value of the parameters $K_{n}$ and $\alpha$ for coal-bearing deposits of the same type can be taken equal to 1 . Parameter $\beta$ depends on the pore space structure and varies in the range $\beta$ $=1.3-2.2$ for compacted non-cohesive soils; parameter $\gamma$ is determined by the degree of the solid body surface wettability and varies in the range $\gamma=1.8-3.5$.

The ranges of values of the parameters $\beta$ and $\gamma$ were obtained by reverse calculations using the experimental $\rho_{\mathrm{K}} / \rho_{\mathrm{B}}$ data bank, as well as the averaged values of the physical and engineering parameters $\mathrm{m}$ and $\mathrm{W}$ for the main types of Kuzbass clayey soil. 
Table 1. Predicted values of structural parameters $\beta$ and $\gamma$.

\begin{tabular}{|c|c|c|c|c|c|c|c|c|c|c|}
\hline \multirow{2}{*}{\multicolumn{2}{|c|}{ Type of soil }} & \multicolumn{9}{|c|}{ Parameter $\boldsymbol{\beta}$} \\
\hline & & \multicolumn{3}{|c|}{$\begin{array}{l}\text { Clay sands }(m=0.314 \\
\qquad W=0.187)\end{array}$} & \multicolumn{3}{|c|}{$\begin{array}{c}\text { Loams } \\
(m=0.296 ; \\
W=0.227)\end{array}$} & \multicolumn{3}{|c|}{$\begin{array}{c}\text { Clays } \\
(m=0.324 ; \\
W=\mathbf{0 . 1 8 9})\end{array}$} \\
\hline \multicolumn{2}{|c|}{ Parameter $\gamma$} & 1,80 & 2.20 & 2.60 & 1.80 & 2.20 & 2.60 & 1.80 & 2.20 & 2.60 \\
\hline \multirow{5}{*}{$\rho_{\kappa} / \rho_{\mathrm{B}}$} & 10.0 & 0.59 & 1.15 & 1.72 & 0.30 & 0.79 & 1.27 & 0.62 & 1.21 & 1.80 \\
\hline & 5.0 & 1.18 & 1.74 & 2.32 & 0.87 & 1.36 & 1.84 & 1.23 & 1.82 & 2.41 \\
\hline & 2.0 & 1.96 & 2.51 & 3.13 & 1.62 & 2.11 & 2.60 & 2.05 & 2.64 & 3.23 \\
\hline & 1.5 & 2.20 & 2.77 & 3.34 & 1.69 & 2.35 & 2.83 & 2.30 & 2.89 & 3.84 \\
\hline & 1.2 & 2.40 & 2.95 & 3.53 & 2.04 & 2.53 & 3.02 & 2.50 & 3.09 & 4.46 \\
\hline
\end{tabular}

To assess the pollutant content in the pore fluid, it is advisable to use the theoretical dependence of the harmonic weighted average for a two-component medium, which will make it possible to calculate the desired value with high reliability:

$$
\frac{1}{\rho_{\mathrm{B}}}=\frac{V_{3}}{\rho_{3}}+\frac{V_{n}}{\rho_{n}}=\frac{V_{3}}{\rho_{3}}+\frac{1-V_{3}}{\rho_{n}}=\frac{V_{3} \cdot \rho_{n}+\rho_{3}-V_{3} \cdot \rho_{3}}{\rho_{3} \cdot \rho_{n}},
$$

where $V_{3}, V_{3}$ - respectively, the volume fractions of the pollutant and natural moisture in the pore filler; $\rho_{3}, \rho_{n}$ - respectively, the effective resistivity of the pollutant and natural moisture.

Theoretical dependencies (1) and (2) in general make it possible to assess the degree of soil pollution based on the results of single measurements, longitudinal and vertical electrical soundings

Further analysis of the process of electrochemical treatment of contaminated soils requires information on the electrophysical properties of natural aqueous solutions and liquids containing pollutants and saturating soils. Experimental studies of the electrical properties of electrolyte solutions containing acids and alkalis with measurements at direct and alternating electric currents have been carried out.

For measurements, a setup consisting of a container for solutions from a non-conductive material was prepared; measurements of the effective electrical resistance (resistivity) at direct and alternating current were made with the help of a four electrode sensor, using a $\mathrm{KP}-1$ logging tool. The distance between contacts $\mathrm{AM}=\mathrm{MN}=\mathrm{NB}=10 \mathrm{~mm}$

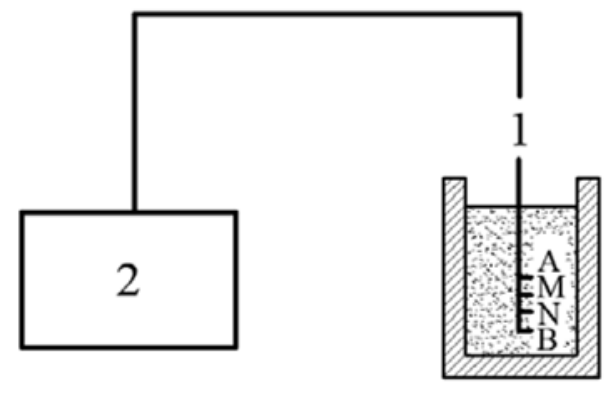

Fig. 1. Measuring setup diagram: 1 - four-electrode sensor; 2 - KP-1 logging tool.

The solution resistivity value was calculated by the formula: 


$$
\rho=\kappa \frac{\Delta U}{I},
$$

where $\mathrm{K}$ - geometry factor of the setup determined from the ratio

$$
\mathrm{K}=\frac{4 \pi}{\frac{1}{\mathrm{AM}}-\frac{1}{\mathrm{AN}}-\frac{1}{\mathrm{BM}}+\frac{1}{\mathrm{BN}}} ;
$$

$\Delta U$ - voltage drop across the electrodes $\mathrm{AN}, \mathrm{V} ; I$ - current through the transmitting electrodes $\mathrm{AB}, \mathrm{A}$.

\section{Results and discussion}

The results of measurements of the resistivity $\rho_{\ni}$ of a $\mathrm{NaCl}$ solution depending on the salt content in the solution and the frequency of the alternating current are shown in Fig. 2. The relationship between the resistivity of acid and alkali from the frequency of alternating current is presented in Fig. 3.; it was found that the resistivity of alkali and acid solutions is in the range of $10^{-3}-10^{-4}$, which indicates their contrast to natural aqueous solutions.

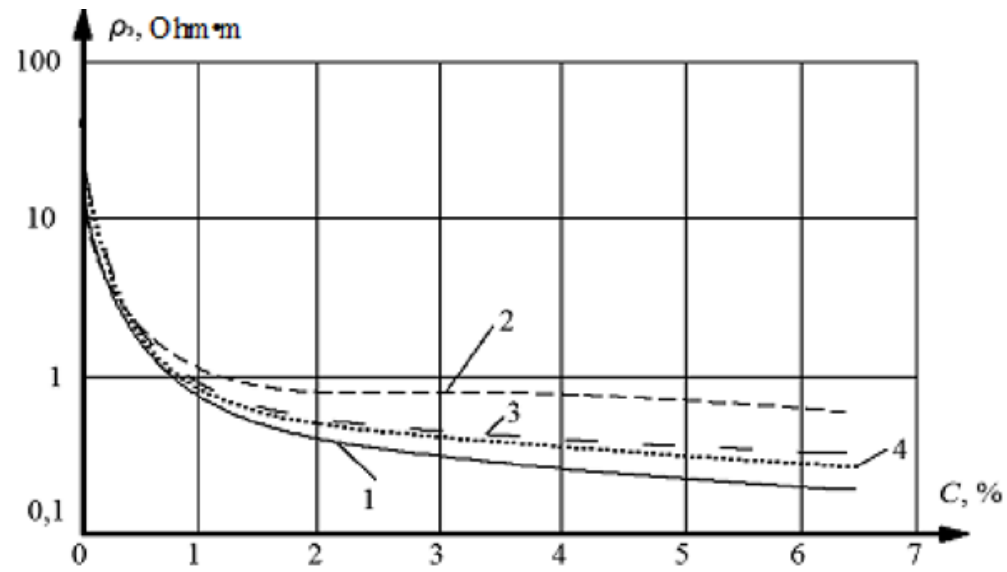

Fig. 2. The dependence of the resistivity of the solution $\rho_{\ni}$ on the salt content $C$ in the solution: 1 direct current; 2 - alternating current, $f=10 \mathrm{~Hz} ; 3-200 \mathrm{~Hz} ; 4-1000 \mathrm{~Hz}$.

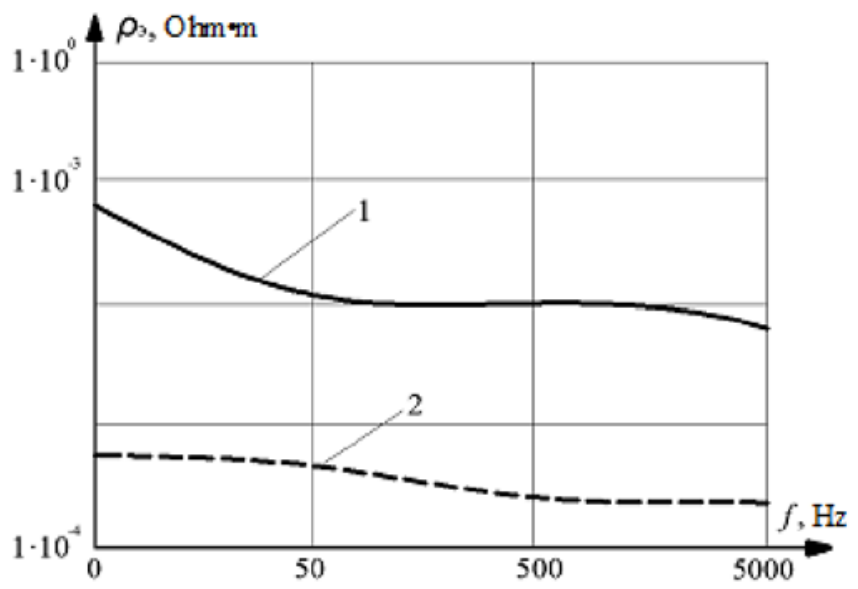

Fig. 3. Dependence of the resistivity $\rho_{3}$ of acid and alkali on the frequency of alternating current: 1 Sulfuric acid $\left(\mathrm{H}_{2} \mathrm{SO}_{4}\right) ; 2-$ Alkali. 
With an increase in the content of acid and alkali in a water solution, a decrease in the resistivity occurs, so that at a content of more than $8 \%$, the resistivity changes insignificantly. The dependences of the absolute $\rho_{3}$ values and resistivity of an acid and alkali solution on their contents are shown in the Figure.
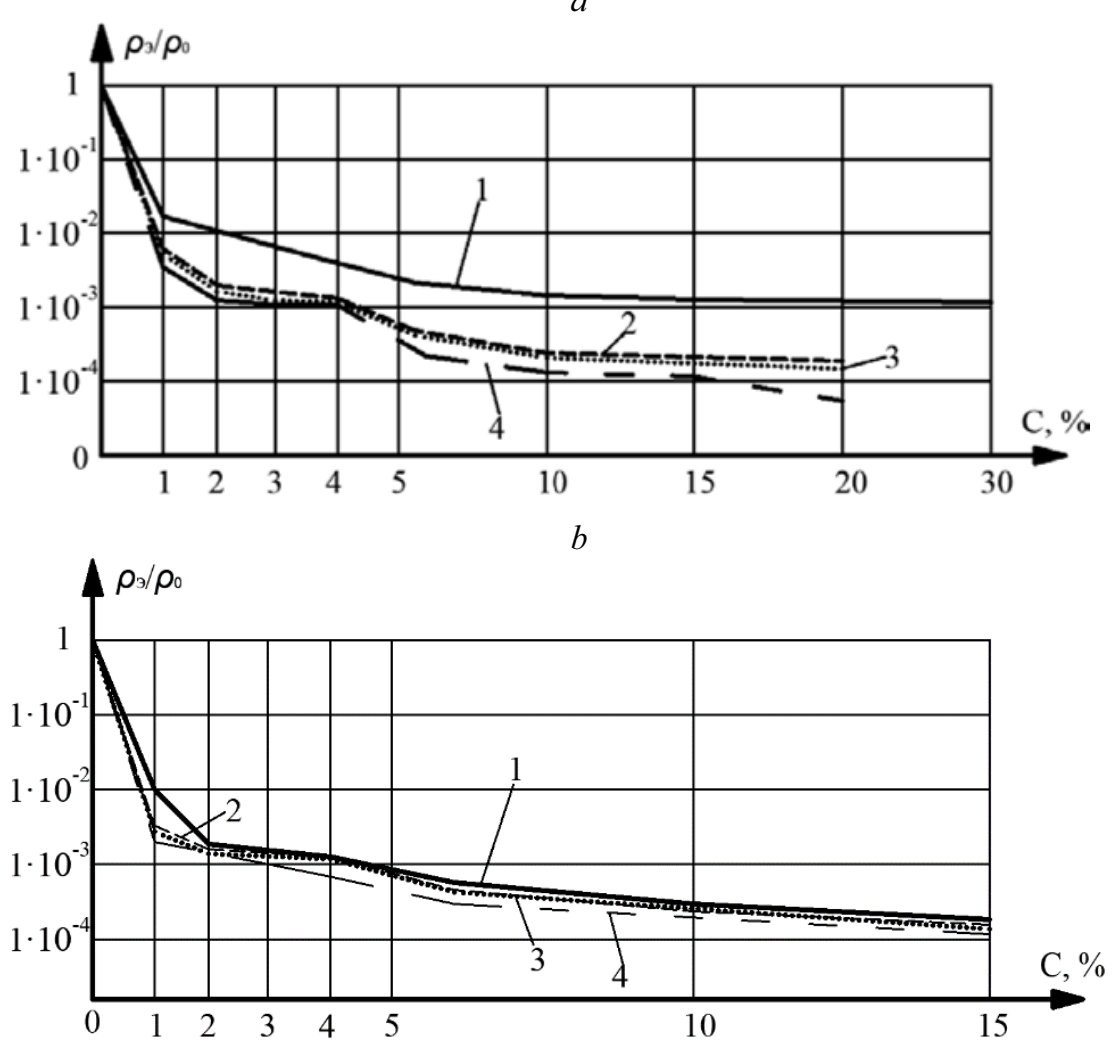

Fig. 4. Dependence of the resistivity of the solution $\rho_{\ni}$ on the acid $(a)$ and alkali $(b)$ content $k$ in the solution: 1 - direct current; 2 - alternating current, $f=50 \mathrm{~Hz} ; 3$ - $500 \mathrm{~Hz} ; 4$ - $5000 \mathrm{~Hz}$.

$a$

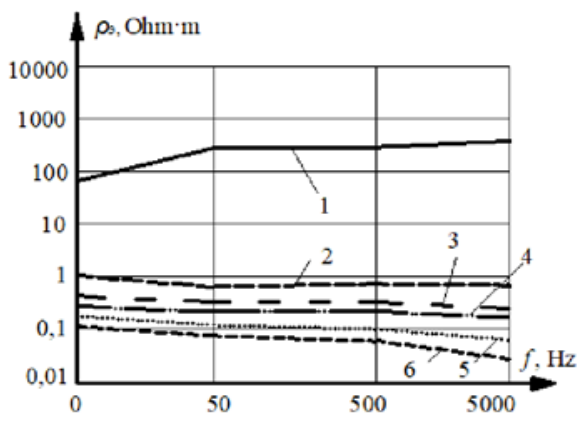

$b$

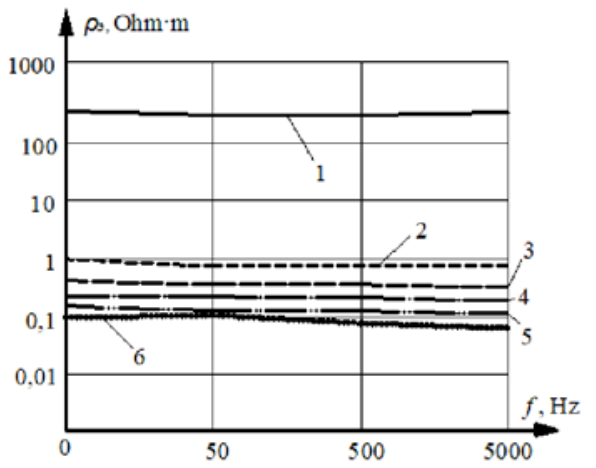

Fig. 5. Dependence of the resistivity $\rho_{3}$ of a solution of water with an acid $(a)$ and an alkali $(b)$ of various contents on the frequency of alternating current: 1 - pure water; $2-k=2 \% ; 3-k=4 \% ; 4-k=$ $6 \% ; 5-k=10 \% ; 6-k=15 \%$. 
By substituting the data of Fig. 4 and Fig. 5 into equation (2), we obtained the dependences of the average $\rho_{6}$ values on the relative content of the pollutant $\mathrm{V}_{3} / \mathrm{V}_{3}$ for constant (Fig. 8) and alternating (Fig. 9) currents.

The above dependences made it possible to form a database for the interpretation of the results of experimental soundings when monitoring the change in the ratio $V_{3} / V_{\ni}$ by the $\rho_{K}$ value.

$a$

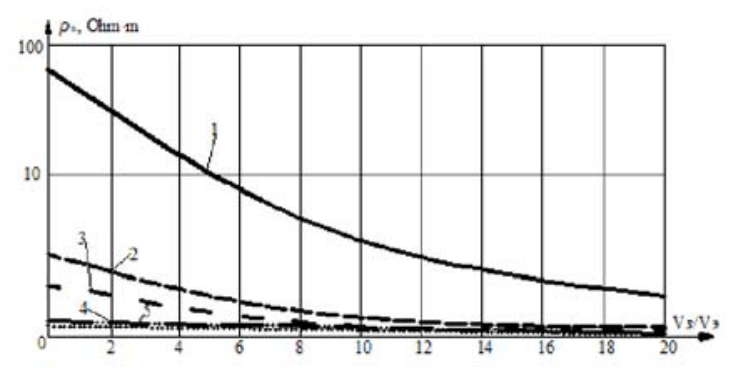

$b$

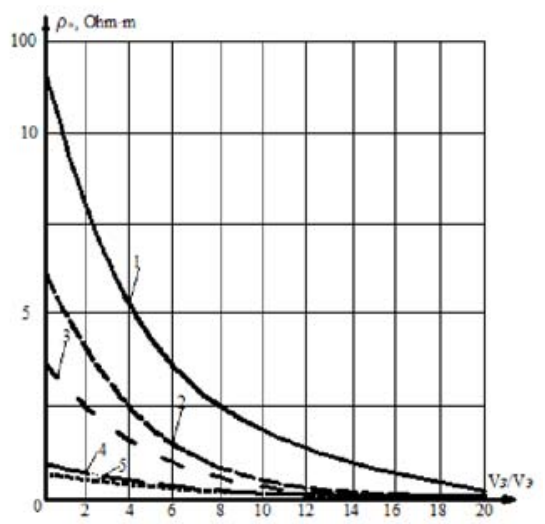

Fig. 6. Dependence of the average $\rho_{6}$ values on the relative acid $(a)$ and alkali $(b)$ content when measured at direct current: 1 - salt content in the solution $C=2 \mathrm{~g} / 1 ; 2-C=5 \mathrm{~g} / 1 ; 3-C=10 \mathrm{~g} / 1 ; 4-C$ $=30 \mathrm{~g} / \mathrm{l}$.

$a$

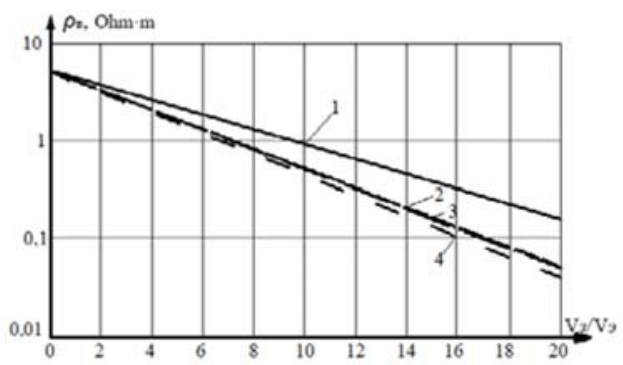

$b$

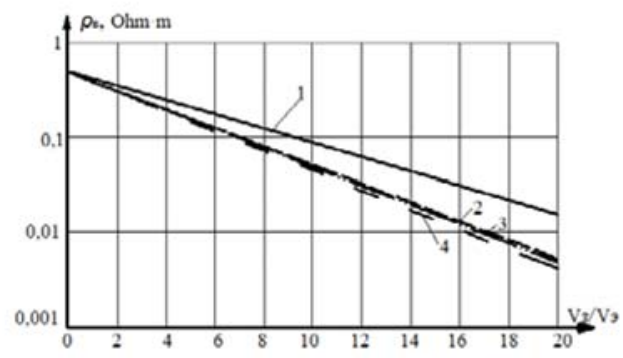

Fig. 7. Dependence of the average resistivity on the frequency of alternating current and the acid $(a)$ and alkali $(b)$ content in the electrolyte at $C=0.2 \mathrm{~g} / 1(\mathrm{a}) ; 3 \mathrm{~g} / 1(\mathrm{~b}): 1-f=0 \mathrm{~Hz} ; 2-50 \mathrm{~Hz} ; 3-500 \mathrm{~Hz}$; $4-5000 \mathrm{~Hz}$.

Taking into account the ranges of structural parameters $\beta$ and $\gamma$, given in Table 1 , the dependences of changes in the effective resistivity of the main clayey soil of the Quaternary deposits of Kuzbass, partially shown in Fig. 8 and 9, were calculated. 

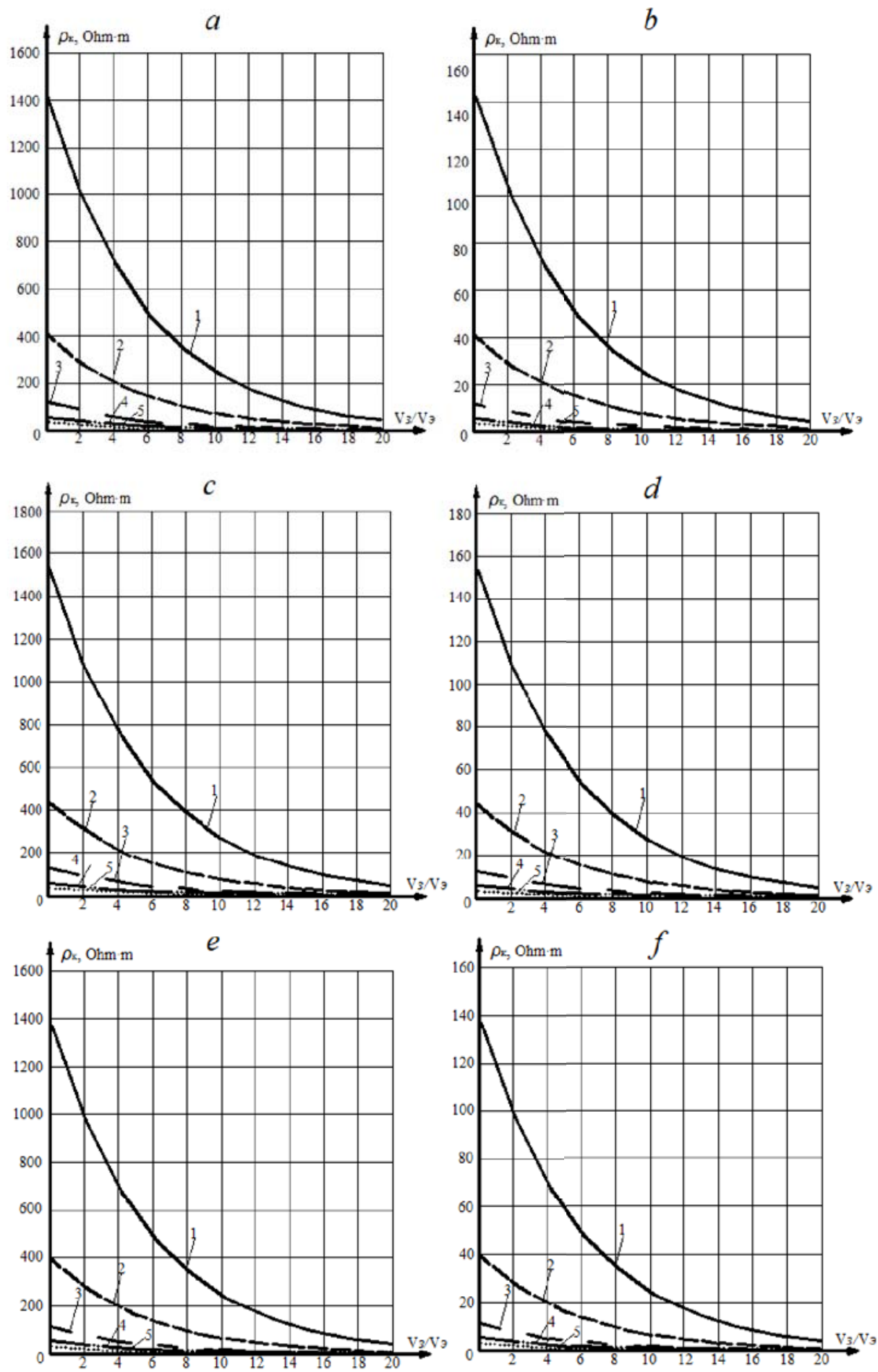

Fig. 8. Dependences of the effective resistivity $\rho \kappa$ of Kuzbass soils on the moisture saturation coefficient of the pore space and cracks $\mathrm{W}$, the relative acid content in the weighted average $V_{\mathrm{H}} / V_{3}$ and the salt content in the electrolyte $\mathrm{C}, \mathrm{g} / \mathrm{l}$ : clay sands $-C=0.2$ (a), $C=3$ (b); loams $-C=0.2$ (c), $\mathrm{C}$ $=3(\mathrm{~g})$; clays $-C=0.2(\mathrm{~d}), C=3(\mathrm{f}): 1-W=0.1 ; 2-W=0.2 ; 3-W=0.4 ; 4-W=0.6 ; 5-W=0.8$. 

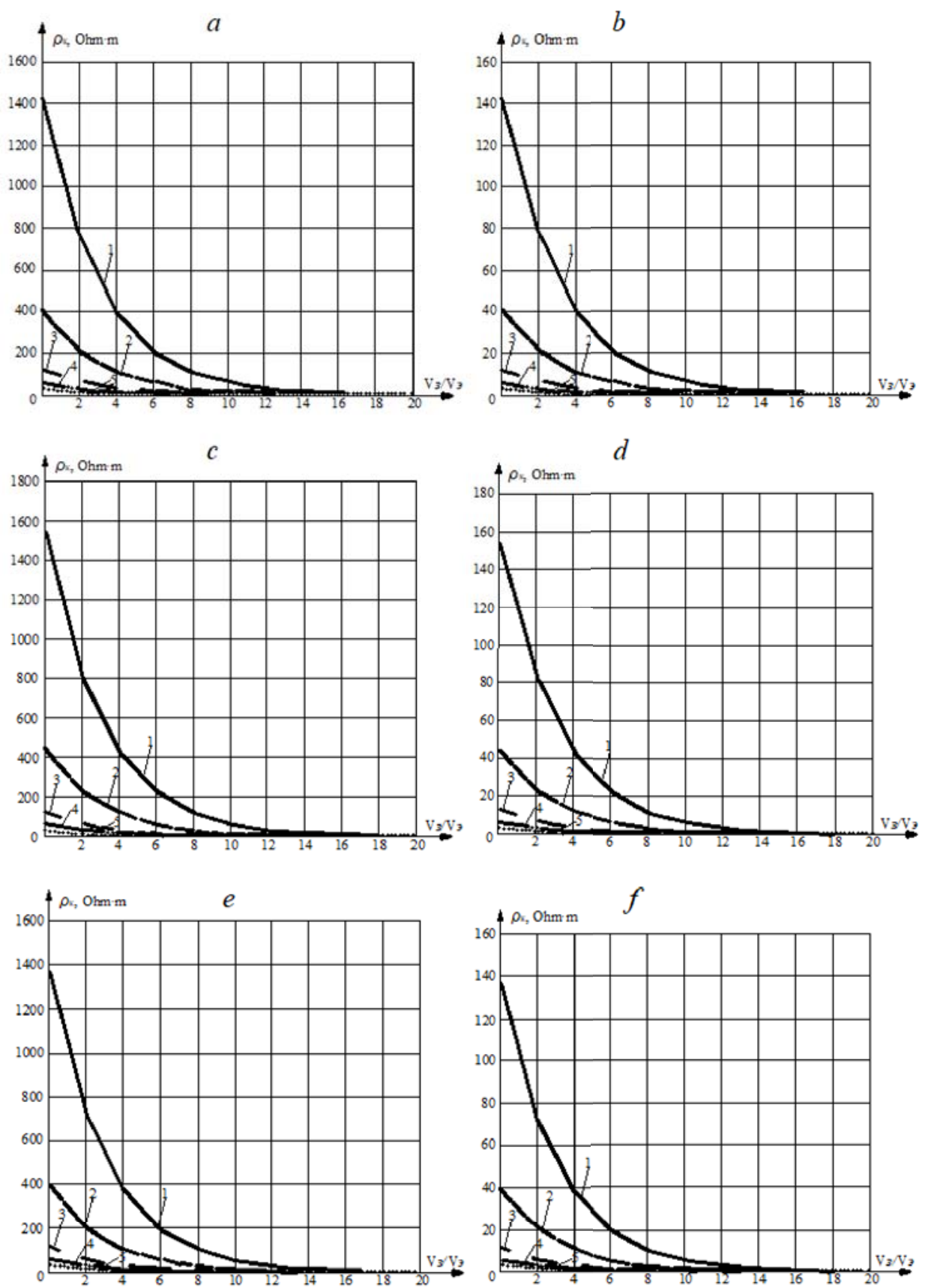

Fig. 9. Dependences of the effective resistivity $\rho \kappa$ of Kuzbass soils on the moisture saturation coefficient of the pore space and cracks $\mathrm{W}$, the relative alkali content in the weighted average $V_{\mathrm{H}} / V_{3}$ and the salt content in the electrolyte $\mathrm{C}, \mathrm{g} / \mathrm{l}$ : clay sands $-C=0.2$ (a), $C=3$ (b); loams - $C=0.2$ (c), $C$ $=3(\mathrm{~g})$; clays $-C=0.2(\mathrm{~d}), C=3(\mathrm{f}): 1-W=0.1 ; 2-W=0.2 ; 3-W=0.4 ; 4-W=0.6 ; 5-W=0.8$. 


\section{Conclusions}

The results of the studies conducted allowed establishing the following:

- at a content of more than $1 \%$, solutions of acids and alkalis are electrically contrasting to natural solutions;

- taking into account the structural parameters of the main types of argillo-arenaceous soils of Kuzbass, a data bank has been collected for diagnosing zones of pollution with inorganic ecotoxicants by negative anomalies of electrical resistivity.

\section{Acknowledgments}

The study was conducted as part of grant MK-1212.2020.5 "Geological and geophysical monitoring of the processes of electrochemical cleaning of soil bases of structures from oil pollution".

\section{References}

1. Ju.Yu. Strelnikova, World of Scientific Discoveries 3(2), 155-167 (2015)

2. A Popova, V. Popova, International Forestry Forum "Forest ecosystems as global resource of the biosphere: calls, threats, solutions" 012047 (2020)

3. S.E. Barlow, M.A. O'Neill, Current Opinion in Insect Science 38, 15-25 (2020)

4. S.L. Turkov, IOP Conference Series: Earth and Environmental Science 012042 (2020)

5. E.G. Kolomyts, Herald of the Russian Academy of Sciences 88(6), 482-493 (2018)

6. D.V. Lopatin, A.I. Zhirov, Geography and Natural Resources 38(1), 30-37 (2017)

7. A. Solovitskiy, O. Brel, N. Nikulin, E. Nastavko, T. Meser, E3S Web of Conferences. The Second International Innovative Mining Symposium (2017)

8. A. Solovitskiy, O. Brel, A. Saytseva, Ph. Kaizer, E3S Web of Conferences. 3rd International Innovative Mining Symposium, IIMS 2018: Electronic edition (2018)

9. Z. Chuncai, L. Guijian, F. Ting, S. Ruoyu, Journal of Geochemical Exploration 143, 54-61 (2014)

10. K.N. Malitch, R.M. Latypov, Mineralogical Magazine 76(6), 2061 (2012)

11. X. Xu, Y. Li, D. Wang, N. Xu, Journal of Fisheries of China 40(5), 731-739 (2016)

12. A.V. Litvinovich, O.Yu. Pavlova, A.V. Lavrishchev, V.M. Bure, E. Salnjikov, Communications in Soil Science and Plant Analysis 52(2), 93-101 (2021)

13. S. Prostov, E. Shabanov, E3S Web of Conferences. The Second International Innovative Mining Symposium 02007 (2017)

14. S. Prostov, E. Shabanov, E3S Web of Conferences. 3rd International Innovative Mining Symposium, IIMS 2018: Electronic edition 02002 (2018)

15. S.M. Prostov, M.B. Gucal, E.F. Shabanov, Taishan Academic Forum - Project on Mine Disaster Prevention and Control, 433-440 (2014) 